\title{
Fiber probe microcavities for refractive index and temperature discrimination
}

\author{
Ricardo M. André*a,b, Stephen C. Warren-Smith ${ }^{\mathrm{b}}$, Martin Becker ${ }^{\mathrm{b}}$, Jan Dellith ${ }^{\mathrm{b}}$, Manfred Rothhardt ${ }^{\mathrm{b}}$, \\ M. I. Zibaii ${ }^{\mathrm{c}}$, H. Latifi ${ }^{\mathrm{c}}$, Manuel B. Marques ${ }^{\mathrm{a}}$, Hartmut Bartelt ${ }^{\mathrm{b}}$, Orlando Frazão ${ }^{\mathrm{a}}$ \\ ${ }^{a}$ INESC TEC and Dep. of Physics and Astronomy, Faculty of Sciences, University of Porto, Rua do \\ Campo Alegre 687, 4150-179 Porto, Portugal; \\ ${ }^{b}$ Leibniz Institute of Photonic Technology (IPHT Jena), Albert-Einstein-Straße 9, 07745 Jena, \\ Germany; \\ ${ }^{c}$ Laser and Plasma Research Institute, Shahid Beheshti University, Evin, Tehran, Iran
}

\begin{abstract}
Fiber probe structures composed of two physical microcavities were created using focused ion beam technology. These structures have a tip-like shape as they were milled in preciously etched tapered fiber tips. The microprobes are then characterized for temperature and refractive index sensing using a signal filtering technique to discriminate signals from distinct microcavities. Using fast Fourier transforms combined with band-pass filters, it is possible to reconstruct the spectra of each cavity independently and thus measure their individual spectral shifts.
\end{abstract}

Keywords: Fabry-Perot interferometer, fiber optics sensors, microstructure fabrication

\section{INTRODUCTION}

Measuring temperature and refractive index is extremely important in many applications. Refractive index is rarely the final parameter to be measured but it is an important stepping stone in creating a bio-sensor ${ }^{1}$. After having a refractive index sensor, one can functionalize said sensor using layers of different materials to create specificity of binding to different chemical and biological species and thus obtain more complex sensing systems. The main problem with temperature and refractive index is that they are rarely independent quantities. Cross-sensitivity is always a problem to overcome when discriminating both quantities.

Several types of fiber sensors have been employed for temperature and refractive index discrimination. Some examples are fiber Bragg gratings (FBGs) ${ }^{2,3}$, long period gratings (LPGs) ${ }^{4}$, a hybrid grating composed of a FBG and $\mathrm{LPG}^{5}$, MachZehnder interferometers ${ }^{6}$ and Fabry-Perot cavities ${ }^{7}$. The most common and simple way to compensate for temperature cross-sensitivity is to use two sensors, one for temperature only and one that measures refractive index (dependent on temperature) $)^{3}$. In most cases, this not only results in the need to read two sensors but also creates another issue: how accurate the temperature measurement is in regards to proximity of both sensors. When measuring in large volumes it may happen that measuring temperature and refractive index a few centimeters apart may have no real impact. This changes when a measurement is needed in a very small volume such as a droplet of blood or even a single cell or organism. That is what this work attempts to solve. The advantage here is the extremely small size of the fiber probes and the proximity of the temperature compensation structure and the refractive index sensing cavity. The fiber probe microcavities are milled with focused ion beam on tapered fiber tip structures. Two contiguous microcavities are created and characterized using a fast Fourier transform (FFT) method combined with signal filtering to achieve temperature and refractive index discrimination.

*rmeloandre@fc.up.pt

Sixth European Workshop on Optical Fibre Sensors, edited by Elfed Lewis, Proc. of SPIE Vol. 9916, 991616 - (C) 2016 SPIE · CCC code: 0277-786X/16/\$18 - doi: 10.1117/12.2236009 


\section{FABRICATION AND CHARACTERIZATION}

Focused ion beam (FIB) technology was used to mill a gap in a tapered fiber tip and to polish the very tip into a reflecting surface ${ }^{8}$. The tapered fiber tip was fabricated by means of dynamic chemical etching, a technique that employs a syringe pump to control the level of hydrofluoric acid when etching the fiber ${ }^{9,10}$. A TESCAN dual-beam FIB/SEM system (Lyra XMU) was used for the milling at $30 \mathrm{keV}$ with ion currents of 1-3 nA for the course milling and 200$400 \mathrm{pA}$ for the final polishing of the reflecting surfaces. The fiber probe structure can be seen in Figure 1 where three cavities are identified.

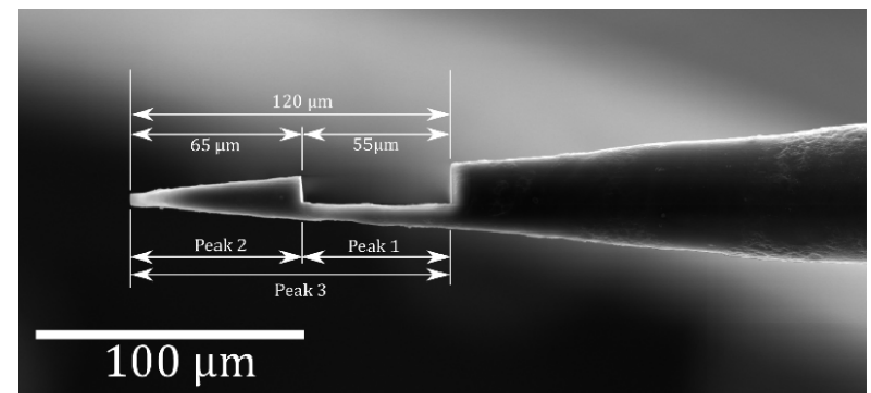

Figure 1. SEM micrograph of the fiber probe structure with a gap cavity and a polished tip.

This probe structure was analyzed in reflection using a setup including a supercontinuum source (Fianium WL-SC-4002), an optical spectrum analyzer (ANDO, AQ6315), and an optical circulator. The obtained spectrum is shown in Figure 2(a). This spectrum clearly presents several interference patterns that are not easily distinguishable. Therefore, a fast Fourier transform (FFT) was performed on the spectrum (w.r.t. optical frequency) to better understand the optical behavior of the structure (see Figure 2(b)).
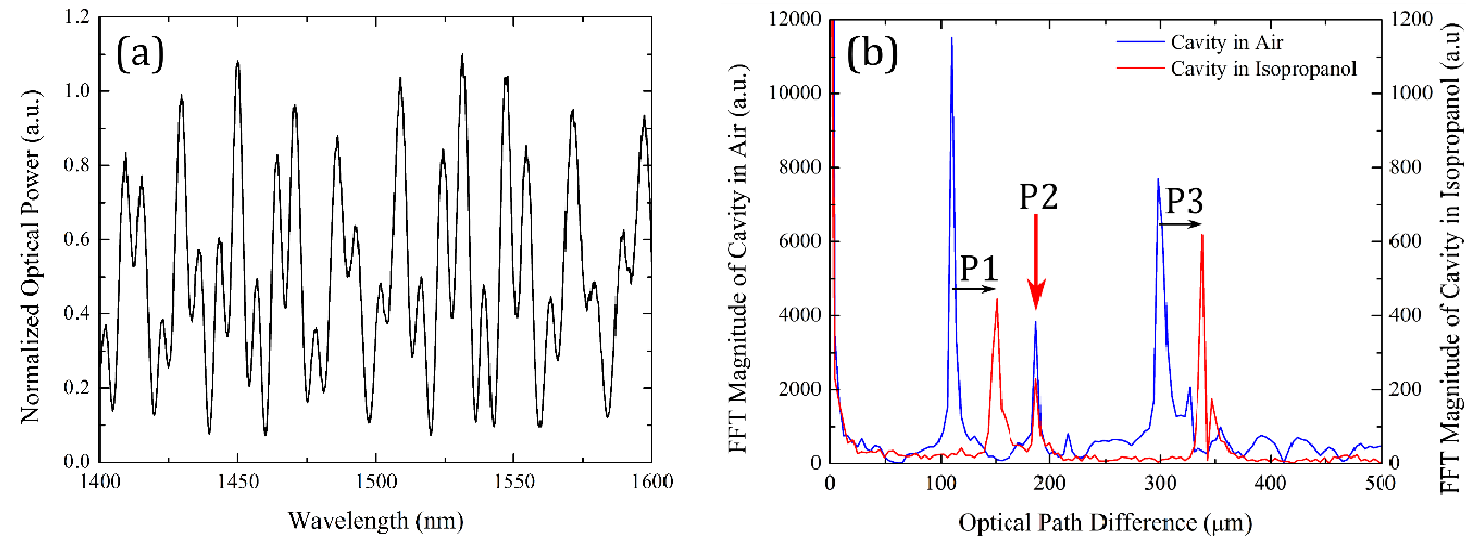

Figure 2. (a) Microprobe spectrum and (b) fast Fourier transforms of said spectrum when the probe is in air (blue) or dipped in isopropanol (red).

In Figure 2(b), two FFT spectra are shown: one where the probe was dipped in isopropanol (IPA) and one where the tip was simply in air. The FFT were performed on the spectrum as a function of wavenumber instead of wavelength to avoid the non-linear spectral dependence on wavelength. Peak 1 originates in the milled gap cavity while Peak 2 results from the silica cavity at the very tip. Peak 3 is a combination of both previous cavities and results from reflections at the first gap interface and at the polished tip.

The FFT spectra show that for Peak 1 and Peak 2 a shift exists that corresponds to the change in refractive index from air to IPA. This shift is the same for both peaks as they both include the exact same gap cavity. Peak 2 does not shift at all, leading to the conclusion that light is confined to the silica and unaffected by the surrounding medium.

\section{RESULTS AND DISCUSSION}

In order to characterize the behavior of each cavity independently, a band-pass filter was applied to the FFT of the signals, to single out each peak. Afterwards, the inverse fast Fourier transform was used to retrieve the spectrum 
resulting from a single cavity. This makes it possible to analyze the contribution of each interferometer independently. In Figure 3(a), several reconstructed spectra for peak 1, when the probe is dipped in water and the water temperature is varied, are shown. A blue-shift is present with increasing temperature. By repeating this analysis in water for the other two peaks as well, the graph shown in Figure 3(b) can be constructed.

The reconstructed spectrum of Peak 2 presents a typical temperature sensitivity of an all-silica Fabry-Perot cavity, further justifying the negligible influence of the external medium on this cavity. The spectra of peak 1 suffer very large shifts due to the refractive index change of water. Peak 3 has an intermediate sensitivity between the two other peaks. The sensitivity of this cavity should be the average of the sensitivities of the two subcavities pondered to the optical path length of each cavity. By taking the sensitivities of Peak 1 and Peak 2 and pondering them to the optical path length, the black (full) line in Figure 3(b) is obtained which is in very good accordance with the experimental data.
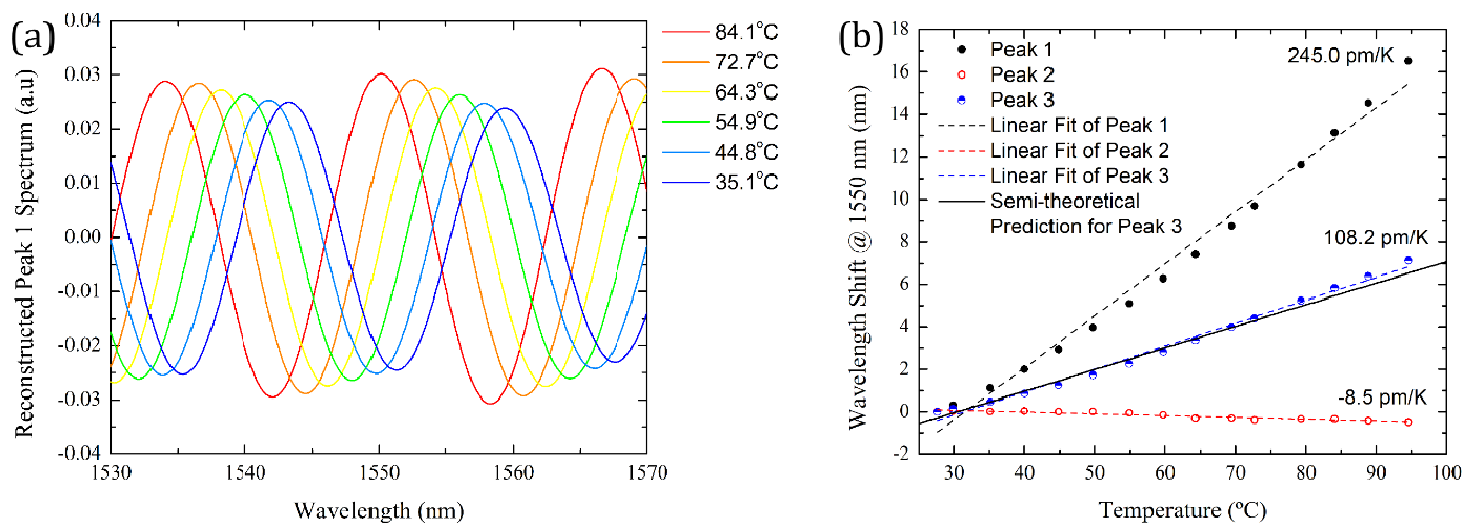

Figure 3. (a) Blue-shift of reconstructed FFT Peak 1 spectra with increasing water temperature and (b) spectral shifts of the reconstructed spectra of each FFT peak with changing water temperature.

By removing the thermal expansion contribution of silica from the response of Peak 1, which is almost negligible, and converting the water temperature into refractive index using calibration curves ${ }^{11}$ it is possible to obtain the results shown in Figure 4.

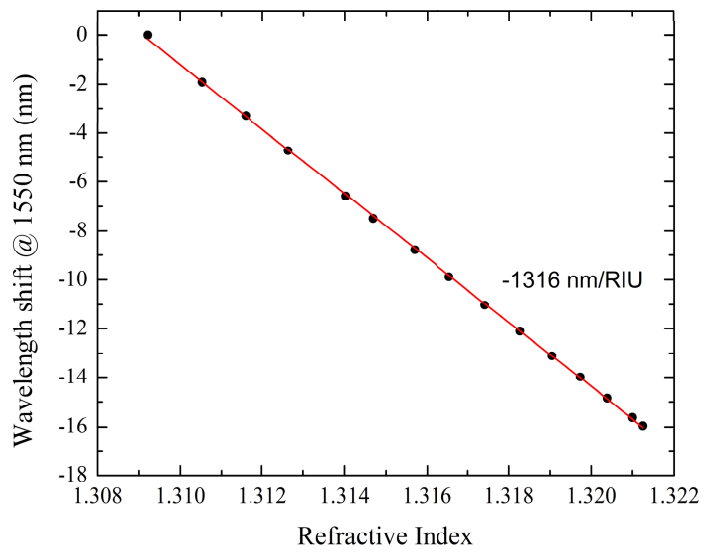

Figure 4. Wavelength shift as a function of refractive index for the reconstructed FFT Peak 1 spectra including a linear fit to the experimental data.

A linear response is obtained for spectral shift dependence on refractive index $\left(r^{2}=0.9998\right)$ with a sensitivity of $1316 \mathrm{~nm} / \mathrm{RIU}$. It is then possible, by combining the data from the FFT peaks, to discriminate temperature and refractive index using these fiber probe microcavities.

\section{CONCLUSIONS}

Even though the sensitivity of this structure is in line with previous refractive index fiber sensors, the advantage of this sensor is that not only is the cavity small (micrometer dimensions) and in a probe-like structure but it also possesses a 
second cavity that is insensitive to refractive index and only sensitive to temperature, allowing for easy compensation. This second temperature sensor is also extremely well located as it is a silica cavity that is immediately besides the refractive index sensor cavity, thus avoiding proximity issues when compensating for temperature.

This microprobe dual-cavity structure allows for temperature and refractive index discrimination in a tip structure with a diameter between 5 and $25 \mu \mathrm{m}$ and a total length of $120 \mu \mathrm{m}$. Further optimization can lead to smaller structures and many potential applications exist. One very interesting application will be to functionalize the gap cavity with a bioindicator and measure in-vivo or in a blood droplet.

\section{ACKNOWLEDGEMENTS}

Project "NORTE-01-0145-FEDER-000036" is financed by the North Portugal Regional Operational Programme (NORTE 2020), under the PORTUGAL 2020 Partnership Agreement, and through the European Regional Development Fund (ERDF). The work of Ricardo André was supported by Fundação para a Ciência e a Tecnologia under the grant $\mathrm{SFRH} / \mathrm{BD} / 84048 / 2012$. Stephen Warren-Smith is supported by the European Commission through the Seventh Framework Programme (FP7), PIIF-GA-2013-623248.

\section{REFERENCES}

[1] Warren-Smith, S. C., Kostecki, R., Nguyen, L. V.., Monro, T. M., "Fabrication, splicing, Bragg grating writing, and polyelectrolyte functionalization of exposed-core microstructured optical fibers.," Opt. Express 22(24), 29493-29504 (2014).

[2] Iadicicco, A., Campopiano, S., Cutolo, A., Giordano, M.., Cusano, A., "Self temperature referenced refractive index sensor by non-uniform thinned fiber Bragg gratings," Sensors Actuators B Chem. 120(1), 231-237 (2006).

[3] Pereira, D. A., "Fiber Bragg grating sensing system for simultaneous measurement of salinity and temperature," Opt. Eng. 43(2), 299 (2004).

[4] Zhang, A. P., "Sandwiched long-period gratings for simultaneous measurement of refractive index and temperature," IEEE Photonics Technol. Lett. 17(11), 2397-2399 (2005).

[5] Chen, X., Zhou, K., Zhang, L.., Bennion, I., "Simultaneous measurement of temperature and external refractive index by use of a hybrid grating in D fiber with enhanced sensitivity by HF etching," Appl. Opt. 44(2), 178 (2005).

[6] Lu, P., Men, L., Sooley, K.., Chen, Q., "Tapered fiber Mach-Zehnder interferometer for simultaneous measurement of refractive index and temperature," Appl. Phys. Lett. 94(13), 131110 (2009).

[7] Wang, T.., Wang, M., "Fabry-Pérot Fiber Sensor for Simultaneous Measurement of Refractive Index and Temperature Based on an In-Fiber Ellipsoidal Cavity," IEEE Photonics Technol. Lett. 24(19), 1733-1736 (2012).

[8] Reyntjens, S.., Puers, R., “A review of focused ion beam applications in microsystem technology," J. Micromechanics Microengineering 11(4), 287-300 (2001).

[9] Haber, L. H., Schaller, R. D., Johnson, J. C.., Saykally, R. J., "Shape control of near-field probes using dynamic meniscus etching.," J. Microsc. 214(Pt 1), 27-35 (2004).

[10] Nikbakht, H., Latifi, H., Amini, T.., Chenari, Z., "Controlling cone angle of the tapered tip fiber using dynamic etching," OFS2014 23rd Int. Conf. Opt. Fiber Sensors, J. M. López-Higuera, J. D. C. Jones, M. López-Amo, and J. L. Santos, Eds., 91574Q, International Society for Optics and Photonics (2014).

[11] Bashkatov, A. N.., Genina, E. A., "Water refractive index in dependence on temperature and wavelength: a simple approximation," Saratov Fall Meet. 2002 Opt. Technol. Biophys. Med. IV, V. V. Tuchin, Ed., 393-395, International Society for Optics and Photonics (2003). 